\title{
RATIO OF MAIN PHYLOTYPES OF GUT MICROBIOTA IN PATIENTS WITH NON-ALCOHOLIC FATTY LIVER DISEASE DEPENDING ON THE BODY MASS INDEX
}

DOI: $10.36740 /$ WLek202103126

\author{
Galyna D. Fadieienko, Nataliia I. Chereliuk, Valentina Yu. Galchinskaya \\ 'GOVERNMENT INSTITUTION “L.T.MALAYA THERAPY NATIONAL INSTITUTE OF THE NATIONAL ACADEMY OF MEDICAL SCIENCES OF UKRAINE”, KHARKIV, UKRAINE
}

\begin{abstract}
The aim: To analyze the status of Gut microbiota (GM) at the level of the main phylotypes in patients with NAFLD, depending on the body mass index (BMI) and gender in comparison with a group of practically healthy individuals.

Materials and methods: The study involved 120 patients with NAFLD, who were divided into two groups depending on BMI and the control group containing 20 practically healthy individuals.

Results: In patients with NAFLD with comorbid obesity, a statistically significant increase in the relative amount of Firmicutes $(52.12$ [42.38; 67.39$] \%)$ and Firmicutes/Bacteroidetes ratio $(3.75[1.7 ; 9.5])$ against the background of a significant decrease in the amount of Bacteroidetes $(13.41[7.45 ; 26.07] \%)$; in NAFLD patients with overweight, the relative amount of Firmicutes was 49.39 [37.47; 62.73]\%, Firmicutes / Bacteroidetes ratio was 1.98 [1.15; 5.92], and the relative amount of Bacteroidetes was 23.69 [12.11; 36.16$] \%$. In the control group, the distribution of the basic GM phylotypes was significantly different; the relative amount of Bacteroidetes was almost the same as of Firmicutes - 34.65 [24.58; 43.53]\% and 29.97 [22.52; 41.75]\% respectively, and the Firmicutes/Bacteroidetes ratio was 0.64 [0.52; 1.47$]$.

Conclusions: The most statistically significant changes in the composition of IM occur due to the increase in the relative amount of Firmicutes and the ratio of Firmicutes/ Bacteroidetes against the background of a decrease in the relative amount of Bacteroidetes. These changes were directly proportional to the increase in BMI, but had no gender features.
\end{abstract}

KEY WORDS: non-alcoholic fatty liver disease, gut microbiota, body mass index

Wiad Lek. 2021;74(3 p.l):523-528

\section{INTRODUCTION}

Non-alcoholic fatty liver disease (NAFLD) is a wide range of liver lesions, which ranges from simple steatosis to steatohepatitis that can progress to liver fibrosis and cirrhosis and transform into hepatocellular carcinoma $[1,2]$.

During the last decades, the incidence of NAFLD has increased worldwide, making it the most common pathology: $25.2 \%$ of the world population is suffering from NAFLD, which is a significant burden for health care $[3,1]$.

In addition, NAFLD is considered to be a polysystemic disease that occurs on the background of concomitant lesions of other organs and systems, including obesity, type 2 diabetes mellitus (T2DM), metabolic syndrome, chronic kidney diseases and cardiovascular disease $[4,5]$.

It is now convincingly proven that gut microbiota (GM) is an important factor of regulation of gastrointestinal homeostasis; its qualitative and quantitative changes can contribute to immune system and metabolism impairments [6].

Increasing evidence suggests that GM is involved in the development of obesity and metabolic syndrome, considering the potential role in the pathogenesis of NAFLD.

However, the data of the conducted studies are ambiguous, which is explained by the influence of numerous genetic and environmental factors.

For instance, when comparing the composition of GM in patients with obesity and in individuals with normal weight or underweight, Ley et al. (2006) $[7,8]$ found relatively low amount of Bacteroidetes and impaired GM diversity in patients with obesity. However, the findings are contrary to many other studies. Duncan et al., (2008) [9], as well as Jumpertz et al., (2011) [10] excluded differences between normal and overweight individuals regarding the relative amount of Bacteroidetes, Firmicutes, or Actinobacteria. Schwiertz et al (2010) concluded in their study that the relative amount of Bacteroidetes increases only in patients with obesity [11].

Despite considerable progress in the study of the status and features of the impact of GM on formation and course of NAFLD, some issues remain insufficiently considered. 


\section{THE AIM}

To analyze the status of GM at the level of the main phylotypes in patients with NAFLD, depending on the body mass index and gender in comparison with a group of practically healthy individuals.

\section{MATERIALS AND METHODS}

The study involved 120 patients with NAFLD. The first group included 89 patients with diagnosed comorbid obesity, whose body mass index (BMI) was 34.50 [31.50; $39.40] \mathrm{kg} / \mathrm{m} 2$ (47 men and 42 women aged from 42.00 to 61.00 years with a median of 49.00 years). The second group included 31 overweight patients with the BMI of 28.00 [27.20; 29.80$] \mathrm{kg} / \mathrm{m} 2$ (17 men and 12 women aged from 36.00 to 56.00 years with a median of 44.00 years).

The control group was formed from 20 practically healthy volunteers, including 8 men and 12 women aged from 37.50 to 55.50 years (median - 50.50 years) with the BMI of 23.50 [21.30; 25.85$] \mathrm{kg} / \mathrm{m} 2$ ). The groups were comparable in age and gender ratio.

In all the examined patients, NAFLD was established by standard procedures. An additional mandatory criterion for inclusion in the study was the presence of excess body weight or obesity. This concomitant pathology was diagnosed using a standard technique - measurement of BMI.

All the patients were interviewed to determine the etiologic factors for the development of secondary liver steatosis and other conditions that affect the intestinal microbiota composition. The energy value and quality of the daily diet and eating habits were estimated using a diet diary that participants completed within 3-7 days.

All the patients underwent general clinical examination (complaint analysis, medical history, life history, objective status) and anthropometry with BMI calculation, assessment of the functional state of liver, carbohydrate and fat metabolism, ultrasound examination of liver.

Blood serum insulin concentration was determined by enzyme-linked immunosorbent assay using DRG reagent kits (Germany) and the NOMA-IR index by the standard formula.

The state of lipid metabolism was analyzed by the level of total cholesterol, low-density lipoprotein cholesterol, triglycerides, and high-density lipoprotein cholesterol. Ultrasound examination of liver, assessment of existence and severity of liver fibrosis was performed using elastometry on Siemens ACUSON S2000 according to conventional methods.

Diagnosis of the bacterial overgrowth syndrome was performed by measuring the changes of concentration of hydrogen in the exhaled air after consumption of $50 \mathrm{~g}$ of glucose (Gastro + Gastrolyzer gas analyzer, UK).

For determination of the basic GM phylotypes, the stool samples collected in sterile containers were aliquoted, rapidly frozen and stored until extraction at $-20^{\circ} \mathrm{C}$. DNA was extracted from $400 \mathrm{mg}$ of stool using the Ribo-prep nucleic acid extraction kit (AmpliSens, RF) according to the manufacturer's instructions.
DNA concentration in the extracts was measured using a Qubit 3 fluorometer with the Qubit dsDNA HS Assay Kits reagents (Thermo Scientific, USA) and equilibrated to 1010 $\mathrm{ng} / \mu \mathrm{L}$. Determination of the composition of GM at the level of basic phylotypes was performed by identification of total bacterial DNA and DNA of Bacteroidetes, Firmicutes, and Actinobacteria by real-time quantitative polymerase chain reaction (PCR) using universal primers for $16 \mathrm{~S} r \mathrm{RNA}$ gene and taxon-specific primers [12].

PCR was performed using the CFX96 Touch Real-Time PCR Detection System (Bio-Rad, USA).

Amplification program:

- the initial stage of denaturation for $5 \mathrm{~min}$ at $95^{\circ} \mathrm{C}-40$ cycles: $15 \mathrm{~s}$ at $95^{\circ} \mathrm{C}, 15 \mathrm{~s}$ at $61.5^{\circ} \mathrm{C}$, and $30 \mathrm{~s}$ at $72{ }^{\circ} \mathrm{C}$ with the reading of fluorescence signal;

- the final stage of elongation $-5 \mathrm{~min}$ at $72{ }^{\circ} \mathrm{C}$.

Statistical processing was performed using Statistica 13.1 package. According to Kolmogorov-Smirnov test, the distribution of all the studied indicators did not meet the normal (Gaussian) one, so the data processing was performed using nonparametric statistics methods. Data are given as Me [LQ; UQ], where Me is the median, and LQ and UQ are the lower and upper quartiles, respectively.

The Bioethics Committee of the State Institution "L.T. Mala National Institute of Therapy of the National Academy of Medical Sciences of Ukraine" approved this study. All the patients were provided with written information regarding the purpose and nature of the study before being included in the study, and they were informed of the possibility of withdrawal from the study at any time without further explanation of their decision. All the examined patients signed a form of informed consent for participation in this study.

\section{RESULTS}

According to the data obtained (tab.1) in both groups of patients with NAFLD, statistically significant changes in anthropometric parameters were noted in comparison with the control group: for instance, an increase of BMI by $46.8 \%$ ( $\mathrm{p}<0.05)$, an increase of waist circumference by $54.2 \%(\mathrm{p}<0.05)$, and an increase of hips circumference by $15.1 \%(\mathrm{p}<0.05)$ was recorded in patients with comorbid obesity. This trend was also observed in the overweight group, though it was less pronounced: BMI was increased by $19.1 \%(\mathrm{p}<0.05)$, waist circumference was increased by $33.3 \%(\mathrm{p}<0.05)$, and hips circumference was increased by $7.9 \%(\mathrm{p}<0.05)$. It should be noted that these indicators were also statistically significant between the first and the second group. The obtained data confirm the abdominal type of obesity typical for patients with NAFLD.

NAFLD is a hepatic manifestation of metabolic syndrome. Its prevalence increases with increasing rates of obesity and insulin resistance, and this statement was also confirmed with our study.

During the study, patients in both groups showed signs of formation of insulin resistance syndrome (for the first group, glycated hemoglobin content was $22.34 \%(\mathrm{p}<0.05)$ 
Table 1. General characteristics of the examined patients

\begin{tabular}{|c|c|c|c|}
\hline \multirow{2}{*}{ Parameter } & \multicolumn{2}{|c|}{$\begin{array}{l}\text { Main group } \\
(n=120)\end{array}$} & \multirow{2}{*}{$\begin{array}{l}\text { Control group } \\
(n=20)\end{array}$} \\
\hline & NAFLD with comorbid obesity $(n=89)$ & NAFLD with overweight $(n=31)$ & \\
\hline \multirow{2}{*}{$\mathrm{BMI}, \mathrm{kg} / \mathrm{m}^{2}$} & $34.50[31.50 ; 39.40]$ & $28.00[27.20 ; 29.80]$ & $23.50[21.30 ; 25.85]$ \\
\hline & $\mathrm{p}<0.05$ & $\mathrm{p}<0.05$ & $\mathrm{p}<0.05$ \\
\hline \multirow{2}{*}{$\begin{array}{l}\text { Waist circumference, } \\
\qquad \mathrm{cm}\end{array}$} & $118.00[108.00 ; 128.00]$ & $102.00[94.00 ; 111.00]$ & $76.50[72.00 ; 80.50]$ \\
\hline & $\mathrm{p}<0.05$ & $\mathrm{p}<0.05$ & $\mathrm{p}<0.05$ \\
\hline \multirow{2}{*}{$\begin{array}{l}\text { Hips circumference, } \\
\text { cm }\end{array}$} & $110.00[105.00 ; 122.00]$ & $103.00[97.00 ; 111.00]$ & $95.50[88.5 ; 101.00]$ \\
\hline & $\mathrm{p}<0.05$ & $\mathrm{p}<0.05$ & $\mathrm{p}<0.05$ \\
\hline \multirow{2}{*}{$\begin{array}{c}\text { Glycated hemoglobin, } \\
\%\end{array}$} & $6.57[5.99 ; 7.36]$ & $6.04[5.43 ; 6.47]$ & $5.37[5.01 ; 5.45]$ \\
\hline & $\mathrm{p}<0.05$ & $\mathrm{p}<0.05$ & $\mathrm{p}<0.05$ \\
\hline \multirow{2}{*}{ HOMA, units } & $5.29[4.06 ; 6.84]$ & $3.80[3.08 ; 4.761]$ & $2.42[1.73 ; 2.97]$ \\
\hline & $\mathrm{p}<0.05$ & $\mathrm{p}<0.05$ & $\mathrm{p}<0.05$ \\
\hline \multirow{2}{*}{$\mathrm{LDL}, \mathrm{mmol} / \mathrm{L}$} & $3.24[2.52 ; 3.87]$ & $2.57[2.05 ; 3.21]$ & $1.26[1.06 ; 1.46]$ \\
\hline & $\mathrm{p}<0.05$ & $\mathrm{p}<0.05$ & $\mathrm{p}<0.05$ \\
\hline \multirow{2}{*}{$\mathrm{TG}, \mathrm{mmol} / \mathrm{L}$} & $1.73[1.17 ; 2.37]$ & $1.40[0.99 ; 2.30]$ & $0.87[0.7 ; 1.12]$ \\
\hline & $\mathrm{p}<0.05^{\mathrm{c} 1 \mathrm{c} 2}$ & $\mathrm{p}<0.05^{\mathrm{c} 1 \mathrm{c} 2}$ & $p<0.05^{c 1 c 2}$ \\
\hline \multirow{2}{*}{$\mathrm{TC}, \mathrm{mmol} / \mathrm{L}$} & $5.43[4.98 ; 6.20]$ & $5.03[4.57 ; 5.38]$ & $4.59[4.05 ; 5.15]$ \\
\hline & $\mathrm{p}<0.05^{12 c 1}$ & & $\mathrm{p}<0.05^{12 \mathrm{c} 1}$ \\
\hline \multirow{2}{*}{ VLDL, $\mathrm{mmol} / \mathrm{L}$} & $0.81[0.58 ; 1.13]$ & $0.78[0.45 ; 1.67]$ & $0.65[0.39 ; 0.77]$ \\
\hline & $\mathrm{p}<0.05^{\mathrm{c} 1}$ & & $\mathrm{p}<0.05^{\mathrm{c} 1}$ \\
\hline \multirow{2}{*}{ ALT, U/L } & $29.00[23.00 ; 42.00]$ & $22.00[18.00 ; 32.00]$ & $17.5[16.00 ; 20.00]$ \\
\hline & $\mathrm{p}<0.05$ & $p<0.05$ & $p<0.05$ \\
\hline \multirow{2}{*}{ GGT, U/L } & $22.30[13.90 ; 41.60]$ & $20.00[13.10 ; 29.00]$ & $17.70[16.20 ; 19.85]$ \\
\hline & $\mathrm{p}<0.05^{\mathrm{c} 1}$ & & \\
\hline
\end{tabular}

Note. $p<0,05$ - statistically significant changes between all the studied groups;

$\mathrm{p}<0,05^{\mathrm{cl}}$ — statistically significant changes in the first group compared with the control group;

$p<0,05^{1122}$ - statistically significant changes in the first and the second group compared with the control group;

$\mathrm{p}<0,05^{12 c 1}$ - statistically significant changes in the first group compared with the control group and statistically significant changes between the first and the second group.

higher, and the NOMA index was 2.19 times higher $(\mathrm{p}<0.05)$ than in the control group; for the second group, it was $12.48 \%(\mathrm{p}<0.05)$ higher and 1.6 times $(\mathrm{p}<0.05)$ higher, respectively).

Lipid metabolism was also analyzed.

Patients in both groups showed a statistically significant increase in low-density lipoprotein (LDL) content compared with the control group - by 2.57 times $(\mathrm{p}<0.05)$ in the first group and by 2.03 times $(\mathrm{p}<0.05)$ in the second group, and by 1.26 times when comparing the first and the second group $(\mathrm{p}<0.05)$. Triglycerides $(\mathrm{TG})$ concentrations in the NAFLD groups also significantly exceeded those of the control group: 1.99 times $(\mathrm{p}<0.05)$ for the first group and 1.6 times $(\mathrm{p}<0.05)$ for the second group; however, it was a trend when the first and the second group were compared.

Total cholesterol (TC) concentrations were statistically different in patients with comorbid obesity by $18.30 \%$ $(\mathrm{p}<0.05)$ and by $7.95 \%(\mathrm{p}<0.05)$ compared with the overweight group. Changes in TC values in the second group 

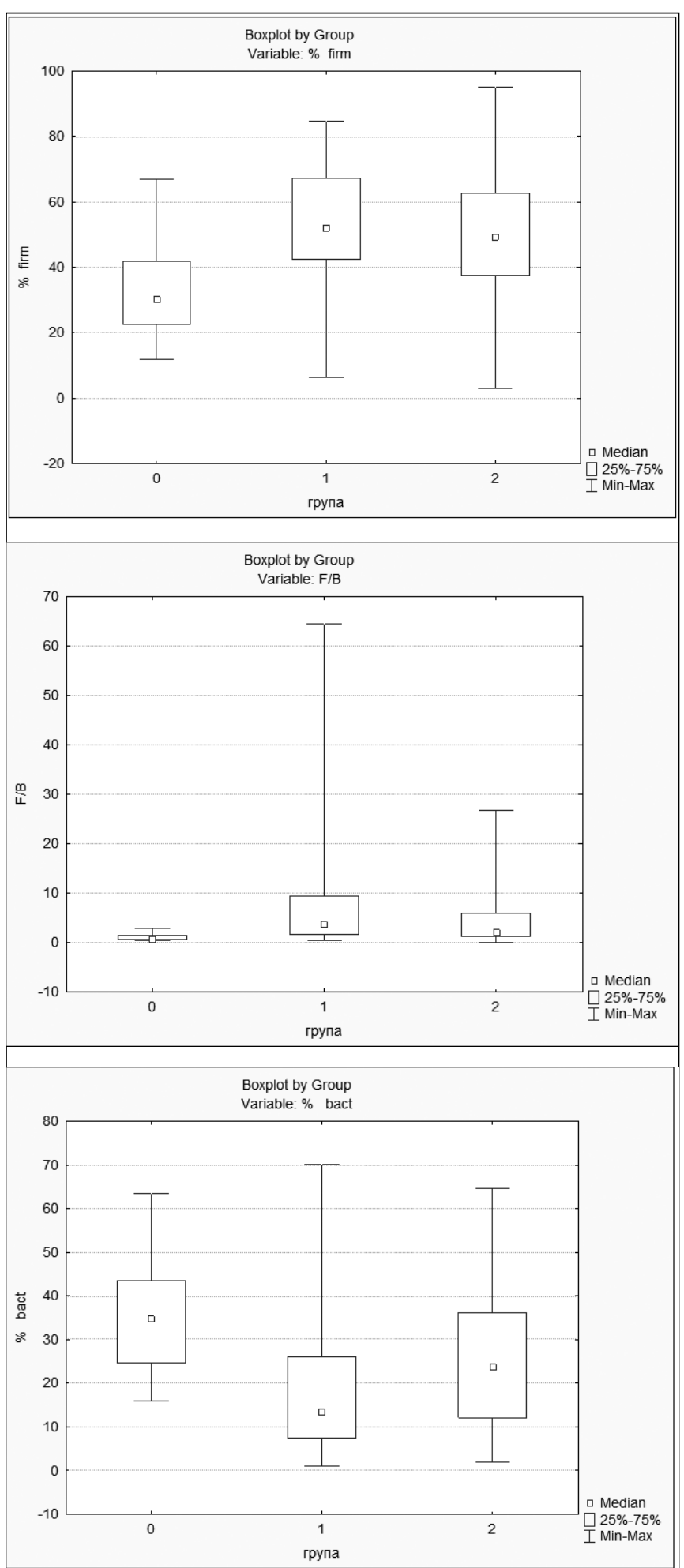

Fig 1. Relative composition main phylotypes of GM in examined patients

when compared to the control group were typical. Very low-density lipoprotein (VLDL) was statistically different only for patients in the first group compared with the control group.

Changes were also noted in parameters showing the functional state of liver: a statistically significant increase by $65.71 \%(\mathrm{p}<0.05)$ in the level of alanine aminotransferase (ALT) and gamaglutamyltranspeptidase (GGT) - by
25.99\% ( $\mathrm{p}<0.05)$ in patients with comorbid obesity; this pattern was observed in overweight patients, but less pronounced for ALT concentrations: by $25.71 \%$, and for GGT it was typical.

A comparative analysis of GM of patients with NAFLD depending on BMI and patients from the control group with normal body weight was performed (fig.1).

The study of relative quantitative composition of GM in the studied groups revealed a number of group dependencies (Kruskall-Wallis test) - namely, in patients with NAFLD with comorbid obesity, a statistically significant increase in the relative amount of Firmicutes (52.12 [42.38; 67.39]\%) and Firmicutes/Bacteroidetes ratio (3.75 [1.7; 9.5]) against the background of a significant decrease in the amount of Bacteroidetes (13.41 [7.45; 26.07]\%), while in NAFLD patients with overweight this tendency was observed only for the relative amount of Firmicutes (49.39 [37.47; 62.73]\%), and Firmicutes/Bacteroidetes ratio was slightly lower $(1.98[1.15 ; 5.92])$ due to increase of the amount of Bacteroidetes (23.69 [12.11;36.16]\%). However, changes in the relative composition of the intestinal microbiota in both the first and the second group were significantly different from the control group by all parameters: Firmicutes - 29.97 [22.52; 41.75]\%, Bacteroidetes - 34.65 [24.58; 43.53]\%, and the ratio Firmicutes/Bacteroidetes amounted to 0.64 [0.52; 1.47]. It should be noted that the relative amount of Actinobacteria, was similar in all the groups: 5 [2.87; 9.14]\%, $4.28[1.53 ; 7.56] \%$ and 5.47 [1.64; $12.79] \%$ according to (pict.2).; the recorded general trend corresponds to our previous results in a smaller study group $(\mathrm{n}=82)$ [13].

\section{DISCUSSION}

Importance of GM in energy homeostasis and nonspecific inflammation, which probably contributes to the development of obesity and metabolic diseases, has been currently proven $[14,15]$.

Recent studies suggest a possible correlation between human GM and obesity. It has been suggested that the Firmicutes/Bacteroidetes ratio may play a role in the development of obesity [16].

Our study findings indicate that GM composition in patients with NAFLD on the background of obesity or overweight (considering the age and gender) may depend on BMI.

The study indicates that the ratio of Firmicutes/Bacteroidetes, which is of great importance in the development of obesity, may vary with BMI. On the one hand, this confirms the data obtained in previous studies, and on the other hand, it refutes them: some authors claim that intestinal dysbiosis develops with NAFLD regardless of the presence of concomitant obesity and/or insulin resistance [17].

These findings were based on the results of a prospective cross-sectional study involving patients with histologically confirmed NAFLD $(\mathrm{n}=39)$, including 15 patients with steatosis, 24 participants with NASH, and 28 healthy individuals. The authors have shown that against the background of NAFLD, the amount of Lactobacillus and Lac- 


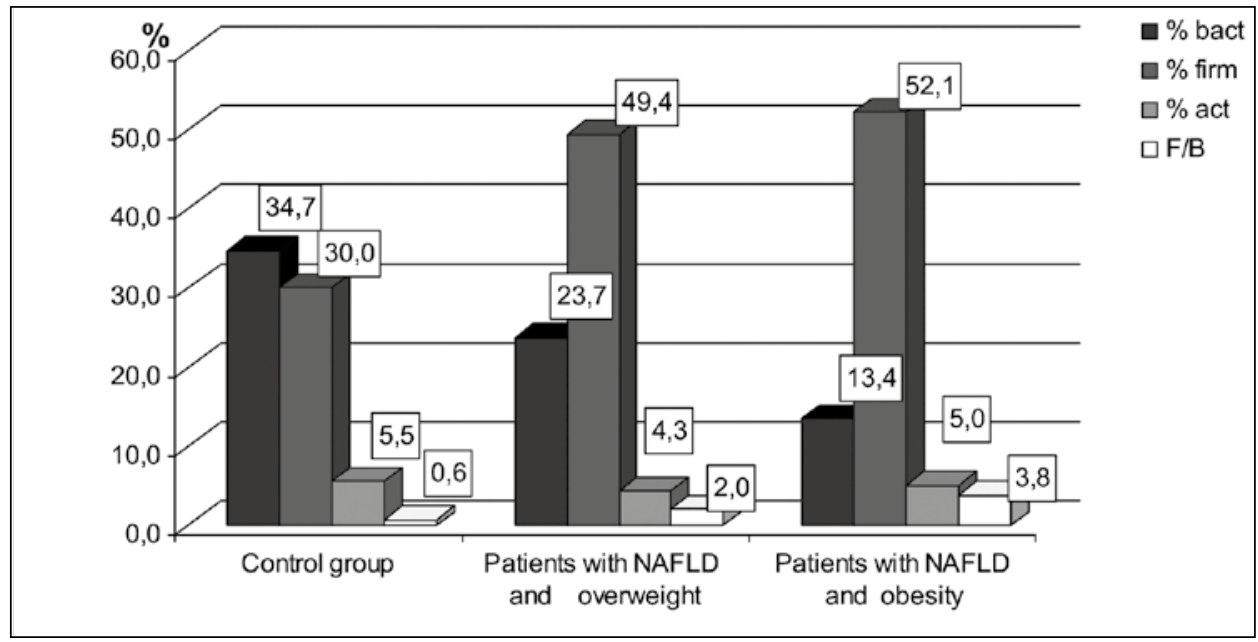

Fig 2. Relative composition main phylotypes of GM in control group (0), patients with comorbid course NAFLD and obesity (1), and patients with NAFLD and overweight (2): firm. - Firmicutes, $\%$; bact. - Bacteroidetes, \%; Firmicutes/ Bacteroidetes- F/B tobacillaceae increased compared to controls. Regardless of the stage of NAFLD, body weight, presence / absence of insulin resistance, scientists noted a decrease in the population of Ruminococcus, Faecalibacterium prausnitzii, and Coprococcus [17].

Researchers from California also refute the correlation between the Firmicutes/Bacteroidetes ratio [18] and agree with the [19] study findings, claiming that the differences in the Firmicutes/Bacteroidetes ratio are much greater among the studies than between individuals with normal weight and overweight in any study.

Moreover, the researchers had initially analyzed the quantitative association of BMI and Bacteroidetes/Firmicutes ratio, determining the relative abundance of the five major bacterial types in each sample, and had made a composite profile at the type level for each individual. No communication was found as a result of the performed work. They then had considered the existence of an association of BMI with a more accurate taxonomic composition, without identifying significant associations, confirming the study performed by Smith MI et al. [20].

The scientists had also analyzed the hypothesis that BMI depends not on the amount, but on the diversity of taxons; however, they refuted this hypothesis, unlike their colleagues Turnbaugh et al. [8] and Le Chatelier et al. [21], who confirmed it.

No gender or age peculiarities were revealed in the ratio of the main GM phylotypes in the patients examined by us.

However, some studies reported differences in the composition of GM in terms of gender. For instance, in the study performed by Haro et al. [22], in patients with a BMI of 23.44 to $41.88 \mathrm{~kg} / \mathrm{m}^{2}$, it was found that women had a lower relative amount of Bacteroidetes compared to men. It should be noted that this study also observed an increase in the Firmicutes/Bacteroidetes ratio, which varied with increasing BMI. However, in a similar study conducted earlier, no differences in GM depending on BMI were found; this may have been due to the fact that only patients with normal and excess weight, and no patients with obesity, were included in the study. In addition, a lower amount of Bacteroidetes was observed in women [23].
Our results have shown that the composition of GM at the level of the main phylotypes differs in individuals with and without obesity, which suggests that these changes are related to body mass and are typical for the studied Ukrainian population.

However, it is difficult to draw any conclusions about the importance of different groups of bacteria in obesity, since not all influential parameters, such as diet, genetic background, race, and living conditions, have been taken into account.

\section{CONCLUSIONS}

The data obtained by PCR method in qRT-PCR mode using universal primers for the 16S rRNA gene and taxon-specific primers from 140 Ukrainians show the most statistically significant changes in the composition of GM by means of increase of the relative amount of Firmicutes and the Firmicutes/Bacteroidetes ratio on the background of decrease of the relative amount of Bacteroidetes. These changes were directly proportional to the increase in BMI, but had no gender features.

In patients with NAFLD, regardless of BMI, a significant violation of enzymatic, lipid, carbohydrate metabolism and changes in the state of insulin resistance were determined, which testifies to the pathogenetic role of metabolic disorders in the development of NAFLD.

Both parallels and differences in the composition of GM associated with both NAFLD and BMI were identified between our study and other studies.

The obtained data confirm the essential role of GM in the pathogenesis of NAFLD. Therefore, it is important to continue the study of the composition of GM at a grandscale level, which in future can serve as a good prognostic marker in the diagnosis and treatment of NAFL in patients with obesity or excess weight.

\section{REFERENCES}

1. Kim D., Siddique 0., Perumpail B.J., Ahmed A. Clinical epidemiology of NAFLD. In Clinical Epidemiology of Chronic Liver Diseases. Springer International Publishing: Cham, Switzerland. 2019; 211-227. 
2. Younossi Z.M., Koenig A.B., Abdelatif D. et al. Global epidemiology of nonalcoholic fatty liver disease-Meta-analytic assessment of prevalence, incidence, and outcomes. Hepatology. 2016;64:73-84.

3. Kim D., Chung G.E., Kwak M.S. et al. Body fat distribution and risk of incident and regressed nonalcoholic fatty liver disease. Clin. Gastroenterol. Hepatol. 2016;14:132-138.e4.

4. Bae J.C., Han J.M., Cho J.H. et al. The persistence of fatty liver has a differential impact on the development of diabetes: The Kangbuk Samsung Health Study. Diabetes Res. Clin. Pract. 2018;135:1-6.

5. Sinn D.H., Cho S.J., Gu S. et al. Persistent nonalcoholic fatty liver disease increases risk for carotid atherosclerosis. Gastroenterology. 2016;151: 481-488.

6. Sunkara T., Rawla P., Ofosu A., Gaduputi V. Fecal microbiota transplant A new frontier in inflammatory bowel disease. J. Inflamm. Res. 2018;11: 321-328.

7. Ley R.E., Turnbaugh P.J., Klein S., Gordon J.I. Human gut microbes associated with obesity. Nature. 2006; 444: 1022-1023.

8. Turnbaugh P.J., Ley R.E., Mahowald M.A. et al. An obesityassociated gut microbiome with increased capacity for energy harvest. Nature. 2006; 444 :1027-1031. doi: 10.1038/nature05414.

9. Duncan S.H., Lobley G.E., Holtrop G. et al. Human colonic microbiota associated with diet, obesity and weight loss. Int J Obes (Lond). 2008;32(11):1720-4. doi: 10.1038/ijo.2008.155.

10. Jumpertz R., Le D.S., Turnbaugh P.J. et al. Energy-balance studies reveal associations between gut microbes, caloric load, and nutrient absorption in humans. Am J Clin Nutr. 2011;94(1):58-65. doi: 10.3945/ ajcn. 110.010132 .

11. Schwiertz A. Microbiota and SCFA in lean and overweight healthy subjects. 2010;18:190-195. doi: 10.1038/oby.2009.167.

12. Turnbaugh P. J. A core gut microbiome in obese and lean twins Nature. 2009; 457:480-484. doi: 10.1038/nature07540.

13. Chereliuk N.I. Ratio of the main phylotypes of gut microbiota in patients with non-alcoholic fatty liver disease and obesity. Modern Gastroenterology. 2019;5(109): 26-33.

14. Backhed F., Ding H., Wang T. et al. The body as an environmental factor that regulates fat storage. Proc. Natl. Acad. Sci. 2004.

15. Turnbaugh P. J., Hamady M., Yatsunenko T. et al. A core gut microbiome in obese and lean twins. Nature. 2009; 457: 480-484.

16. Bajzer M., Seeley R.J. Physiology: obesity and gut flora. Nature. 2006;444:1009-1010.

17. Finucane M.M., Sharpton T.J., Laurent T. J., Pollard K.S. Taxonomic Signature of Obesity in the Microbiome? Getting to the Guts of the Matter PLoS One. 2014;9(1):e84689. doi: 10.1371/journal.pone.0084689.

18. Arumugam M., Raes J., Pelletier E. et al. Enterotypes of the human gut microbiome. Nature 473: 174-180. Ta The Human Microbiome Project Consortium Structure, function and diversity of the healthy human microbiome. Nature. 2012; 486: 207-214.
19. Smith M.I. et al. Gut microbiomes of Malawian twin pairs discordant for kwashiorkor. Science. 2013;339(6119):548-54. doi: 10.1126/ science. 1229000.

20. Le Chatelier E., Nielsen T., Qin J. et al. Richness of human gut microbiome correlates with metabolic markers. Nature. 2013;500:541-546.

21. Haro A. et al Intestinal Microbiota Is Inuenced by Gender and Body Mass Index Carmen. 2016. https://doi.org/10.1371/journal.pone.0154090.

22. Dominianni C., Sinha R., Goedert J.J. et al. Sex, body mass index, and dietary fiber intake influence the human gut microbiome. PloS one. 2015;10:e0124599.

This work was carried out as part of the planned scientific topics of Government Institution "L.T.Malaya Therapy National Institute of the National Academy of Medical Sciences of Ukraine", "To develop a new technology for the personalized treatment of patients with non-alcoholic fatty liver disease against metabolic disorders» (state registration No. 017U003030).

\section{ORCID and contributionship:}

Galyna D. Fadieienko: 0000-0003-0881-6541 A,E,F

Nataliia I. Chereliuk: 0000-0003-0881-6541 B,C,D

Valentina Yu. Galchinskaya: 0000-0002-0024-131X ${ }^{A, C, E}$

\section{Conflict of interest:}

The Authors declare no conflict of interest.

\section{CORRESPONDING AUTHOR Nataliia I. Chereliuk}

"L.T.Malaya Therapy National Institute of the National Academy of Medical Sciences of Ukraine" 2 a Lyubovi Maloy ave., 61039 Kharkiv, Ukraine tel: +380988614251

e-mail: nat.chereliyk@gmail.com

Received: 29.04 .2020

Accepted: 30.11 .2020

A - Work concept and design, B - Data collection and analysis, C - Responsibility for statistical analysis, $\mathbf{D}$-Writing the article, $\mathbf{E}-$ Critical review, $\mathbf{F}-$ Final approval of the article 